\title{
The Critical Technology Research on Rotary Steering Drilling System
}

\author{
Li XiaoJun \\ Mechanical and Storage Engineering Department, \\ China University of Petroleum, Beijing \\ Changping, Beijing, China \\ e-mail: xjlixiaojun@tom.com \\ Jiang Jiansheng \\ Mechanical and Storage Engineering Department, \\ China University of Petroleum, Beijing \\ Changping, Beijing, China \\ e-mail: johnjjs@sina.com
}

\author{
Jiang Bo \\ Drilling Technology Research Institute Of Xinjiang Petroleum \\ Administration Bureau, \\ Karamay city, Xinjiang, China \\ e-mail: 280042880@qq.com \\ Mu Zongjie \\ Drilling Technology Research Institute of Xinjiang Petroleum \\ Administration Bureau, \\ Karamay city, Xinjiang, China \\ e-mail: muzj20023@163.com
}

\begin{abstract}
In order to overcome the shortcomings of slide steering technology, the rotary steerable drilling technology is a new technology developed, this technique represents the highest level of drilling technology development. At the same time, rotary steerable technology is becoming one of the world-class technical problems, and there are a lot of research scholars at home and abroad. Domestic rotary steerable drilling technology research started late, therefore, developed with our own intellectual property rotary steerable drilling technology can significantly improve our oilfield services company's market competitiveness, and generate enormous economic benefits. The paper focuses on the static thrust against rotary steerable systems, rotary steerable drilling technology on the overall composition, tools and software, When using the rotary steerable drilling technology for drilling operation, it does not require frequent tripping you can achieve a tree-dimensional trajectory control, and this technology not only has greater advantage of smoother wellbore trajectory and extending a distance, but also has important significance for ensuring the well trajectory quality, increase drilling speed and efficiency. And for complex structure wells at present the development of special reservoir needs to have the important meaning. In order to provide technical reference for the development of domestic rotary steerable drilling tool.
\end{abstract}

Keywords-Rotary Steering; Push against the stationary rotary steerable systems; Composition; Tools; Software

\section{SIGNIFICANCE OF THE RESEARCH}

Currently, the maturity technical of rotary steerable drilling is monopolized by some foreign companies, including Schlumberger, Baker-Hughes, Halliburton and so on. They still blocked the domestic implementation of technology, and provided exclusively costly services, so that the corresponding drilling costs increase greatly. Domestic rotary steerable drilling technology research started late, and in the development stage, therefore, to break the foreign monopoly, developed with our own intellectual property rotary steerable drilling technology can significantly improve our oilfield services company's market competitiveness, and generate enormous economic benefits.

When using the rotary steerable drilling technology for drilling operation, it does not require frequent tripping you can achieve a tree-dimensional trajectory control, and this technology not only has greater advantage of smoother wellbore trajectory and extending a distance, but also has important significance for ensuring the well trajectory quality, increase drilling speed and efficiency, demand to meet the complex structure of wells drilled [1-5].

Therefore, this article focuses on the static thrust against rotary steerable; the tool can achieve three-dimensional trajectory control, and has greater advantage of smoother wellbore trajectory and extending a distance. It also has the important significance for ensuring the well trajectory qualities, increasing drilling speed and efficiency and demanding to meet the complex structure of wells drilled.

\section{PUSHES AGAINST THE STATIONARY ROTARY STEERABLE SYSTEMS COMPONENTS}

Rotary steerable drilling systems is composed by the ground control system, ground-underground two-way communication systems, LWD systems, MWD systems power supply systems inclination measurement and control systems-oriented guide the implementing agencies and so on (Fig .1). Depending on the different of system structure, the system is divided into geometric rotary steerable drilling system and geo-steering rotary drilling system, in which geometric steerable drilling system does not contain LWD systems. Rotary steerable drilling tool includes the underground part of ground - underground two-way communication system, power supply system, attitude measurement and orientation control system, guide actuating mechanism.

The monitoring system of ground is an indispensable part in rotary steerable drilling system, and it is the command center of the entire system. 


\section{A. Data Acquisition System}

Downhole upload data include: MWD measurement data, near-bit trajectory parameters and reaction conditions downhole parameters. All data uploaded be used to analyze and judge the work state of downhole tools, and whether the trajectory meet the design requirements. Upload data is received and interpreted by the ground-based data acquisition system, the pulse pressure signal transmitted via downhole pressure sensor mounted on the riser capture, after decoding means decodes sent to ground control computer. The data collected by pressure sensor is sent to the decoding device via a wireless transmitter, wireless transmission instead of cable transmission, That greatly simplifies the installation and maintenance procedures, and to ensure the accuracy of the data transmission. Decoding device can be directly connected to a computer via a communication cable ground monitoring or data transmission lines.

\section{B. Terrestrial Monitoring Software System}

Terrestrial monitoring software system including uploading signal decoding software, track deviation vector calculation and analysis software, download signal coding software. Upload signal

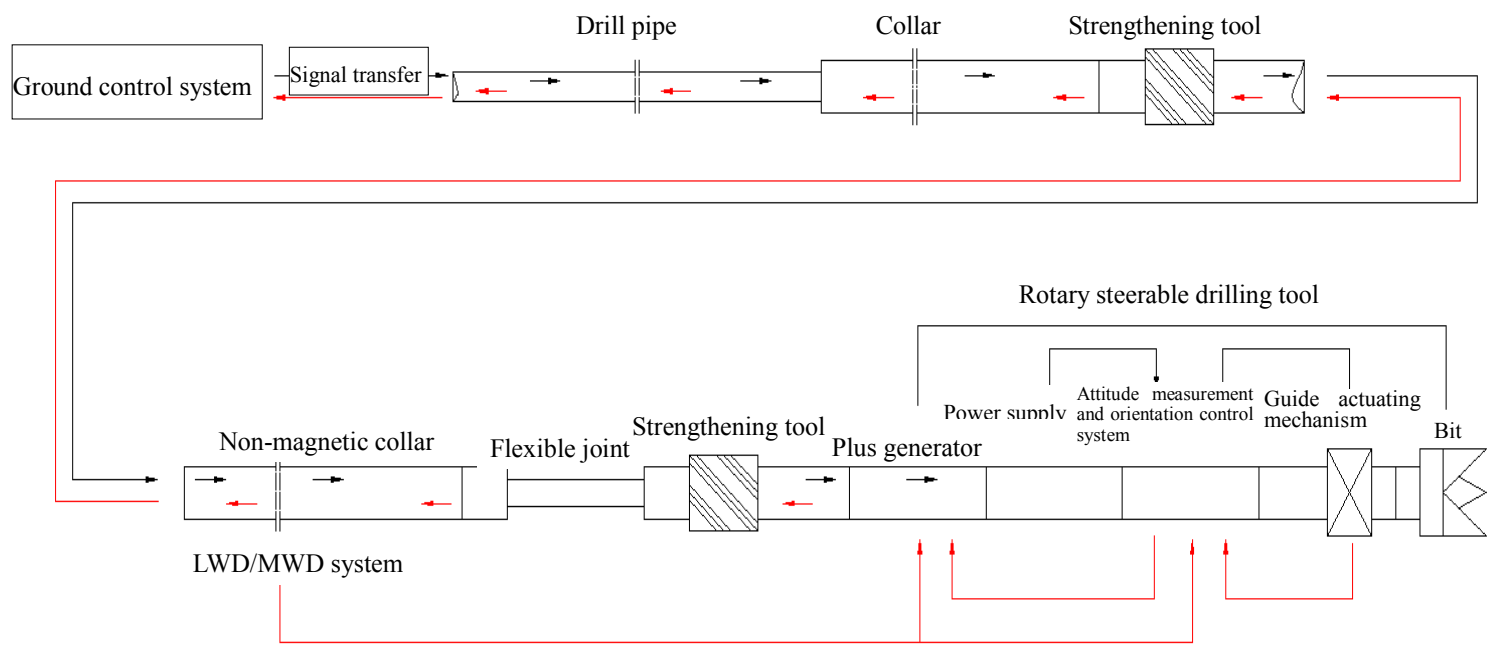

Figure 1. Rotary steerable drilling system constitutes

decoding software receive downhole date that sent by terrestrial decoding, and then was reduced to numerical information readable by ground personnel.

The role of download signal decoding software is to convert the trajectory control command parameter to control instruction encoding. The encoded instruction is transmitted to the downhole tools are implemented through the bypass system. The downlink instruction code is through the opening and closing of the bypass valve (corresponding to the peaks and valleys) and the delay time (corresponding pulse width) between the permutation and combination to achieve, but the coding on the premise that can not affect circulating drilling fluid, upload, download decoding, encoding software is the trajectory deviation vector calculation and analysis software to calculate section.

\section{Bypass system actuators}

Signal download process mainly depends on our ability to adjust the flow in the ground, in this way, the downhole flow can be changed, and then downhole tools can decode according to changes in flow. The flow of ground is adjusted by bypass actuator that is installed on the vertical pipe. This tool can transfer a part of the slurry back to the mud pool.

Bypass actuator is an integrated set up skid, which including gas supply lines, ball valves, union, manifold and other parts. It is used to control the on and off of air supply through a solenoid valve, air from the air compressor on the drilling crew. The opening and closing of valve can control the opening and closing of air supply. When the valve is turned on can be made a part of the mud inside the riser directly back to the mud pools. Close valve position adjustable nozzle installed, played the role of the throttle, you can prevent the valve open all the mud displacement mud through the manifold of bypass actuator directly back to the mud pool, causing mud downhole generator stops working, causing downhole tool does not work normally without power source.

\section{PUSHES AGAINST ROTARY STEERING SYSTEM}

\section{TOOLS COMPONENTS}

\section{A. Data Acquisition System}

The role of two-way communication and power short section is responsible for downhole tools with different data pulse sequence, identification download signal, power generation and pulse control, which include a pulse generator, turbine generator and circuit module mud three parts. As shown in Fig .1, the short section of our design has three drill collar, from top to bottom, the pulse generator is located in the first paragraph of the drill collar, mud turbine generators located within the second stage of the drill collar, circuit module is located within the third drill collars, drill collars which the first section and a second section connection through the drill pipe drill collar buckle, it does not involve docking circuit; the second paragraph and the third paragraph of the drill 
collar to be simultaneous drill buckle docking connections and circuits butt; third lower part of the drill collar to reserve circuit interface to enable two-way communication \& docking power short section with a short section of MWD.

\section{B. short section of the guide structure}

As shown in Fig .2 the main part of a short section of the guide by the rotating mandrel without rotation of the outer cylinder, the guide ribs, lower joint, bearing the TC group, non-contact transmission device, the guide control system, hydraulic module, under TC bearing groups, etc.

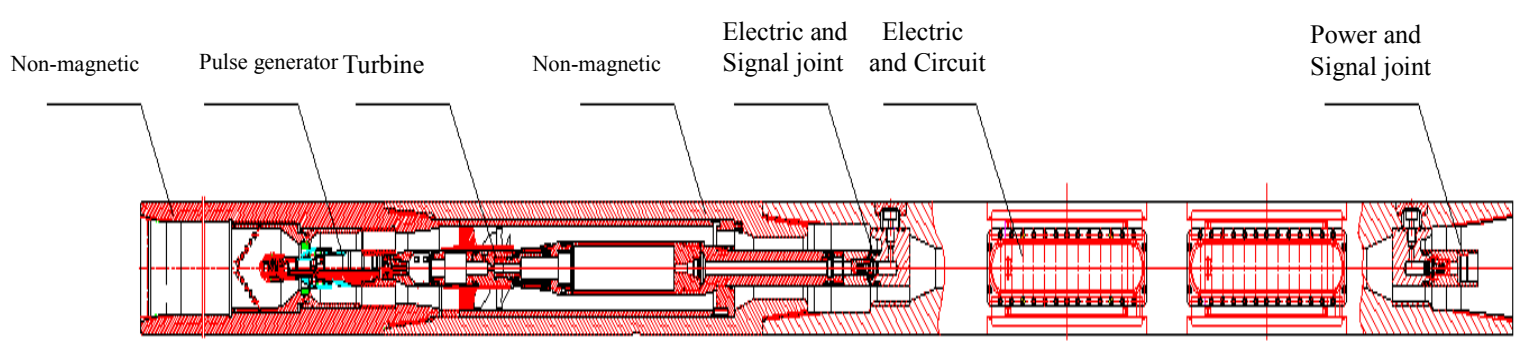

Figure 2. The section of structure bidirectional communication \& power short
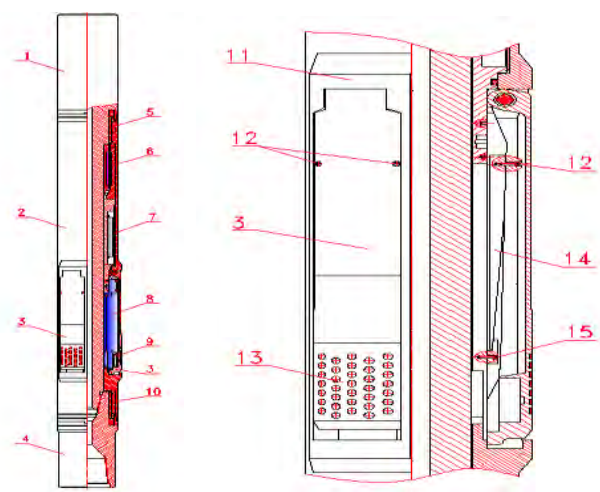

a. Guide short section b. Guide short section enlarged

(Without hydraulic module)

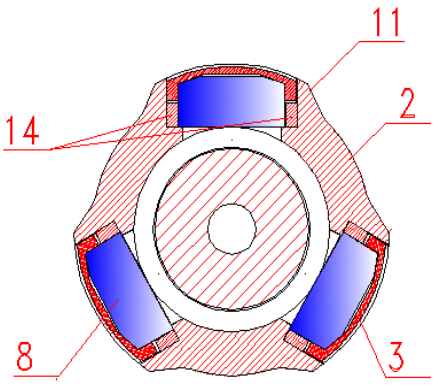

c. Short-sectional view of the guide section

Figure 3. Short guide section and an enlarged view

\section{Guidance control system}

Guidance control system comprises a basal body and a control circuit which is mounted inside the structure. In the middle position of upper annulus between the does not rotate of outer cylinder and the rotation of spindle, has a certain gap with the rotating core shaft, by sealing the system and limiting device clings the non rotating cylinder. The rotating core shaft rotates orientation control system and non rotating outer cylinder core shaft stationary relative rotation. The lower of Guidance control system matrix has three conductive slots and the screw hole, which circumferential direction is distributed. The role of threaded holes is connect the upper of hydraulic and guidance control system matrix. The conductive slot is used connect hydraulic module and the power supply and communication lines guidance control system.

The circuit of control system is installed board slots of guidance control system matrix. It is composed by a main control circuit board, a power management board and three motor-driven plate. Power management board receives electricity that distributed by rotary steerable systems, after conversion, distribution, and then provides differentiated electricity to the hydraulic module motor, hydraulic module sensor, the main control circuit board and motor driver board. Once the rotary steerable force distribution software and trajectory deviation vector analysis software are written inside the main control circuit board, can automatically calculate the trajectory of deviations based on trajectory data from external input or code the corresponding control command based on the ground sent intervention instructions, and then sent to the three motor drive boards. Motor driver board control module and its corresponding hydraulic motor speed, and then control the hydraulic module generates three different fluid pressures. Three hydraulic pressures form the pushes against forces which has specific direction and magnitude. In this process, the motor driver board real-time receives motor feedback signal and the internal pressure of the hydraulic module pressure sensor signal, and then sent the received signal of pressure and speed to the main circuit board by CAN communication. Main circuit board based on feedback pressure and speed information to verify whether the correct instruction issued execution, and a hydraulic module is stable. 


\section{STATIC PUSH-STYLE NAVIGATION CONTROL BY MECHANICAL ANALYSIS}

\section{A. Mathematical Model}

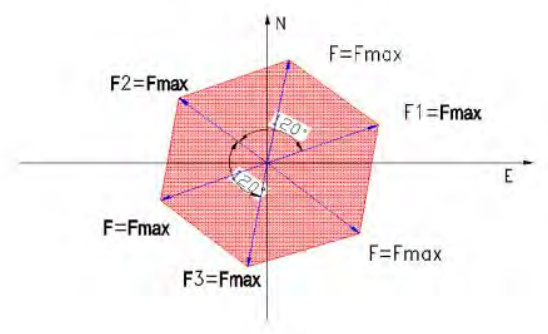

Figure 4. Pushing force force ranges

The magnitude and direction of the guide force is mainly controlled by the control module. If we need to adjust the magnitude and direction of the guide force, the control module just distribute the three hydraulic fluid pressures, and achieve the capacity of guiding the direction and force whip stocking. In generally guide state, the lateral force can be seen from Fig .3. Biasing force of three hydraulic module respectivelyF1,F2,F3 Three vector superposition of force as follows:

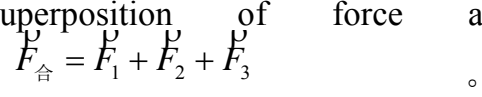
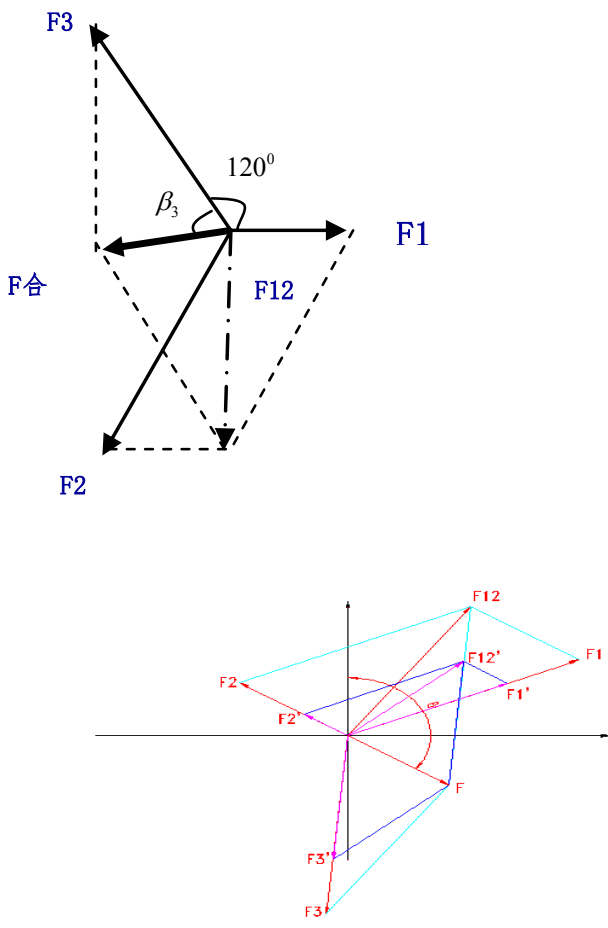

Figure 5. Force Vector Synthesis

In the formation of hard and can provide a large enough support reaction force, the hydraulic system can provide the maximum hydraulic pressure and the piston area under certain circumstances, each guide rib can be obtained the maximum supporting force is Fmax, F1 $\leq$ Fmax, F2 $\leq$ Fmax, F3 $\leq$ Fmax. By mechanical force vector synthesis principle analysis, in three component force any two force reaches the maximum value, and the remaining component value is 0 ,in this case, we can gain the maximum absolute value . Therefore, the absolute value of the Pushing force

$\mathrm{F}$ force falls within the regular hexagon as shown in Fig .5, which is in the range: $0 \leq(\mathrm{F} 1, \mathrm{~F} 2, \mathrm{~F} 3) \leq \mathrm{Fmax}$.

Analysis of the biasing force vector control

The range of the push force vector is a regular hexagon; the guide head shell rotation can be regarded as hexagonal rotation, that shown in Fig .6. Regular six edge rotating can formed inner and outer envelope circle, between the inner and outer envelope circle area, by the force vector synthesis principle known, the direction of push force does not have 360 degrees arbitrary adjustable if the resultant force absolute value remains unchanged, it means the area between the inner and outer circle is called "control dead zone", which should be avoided in the engineering design and directional drilling selection biasing force resultant vector.

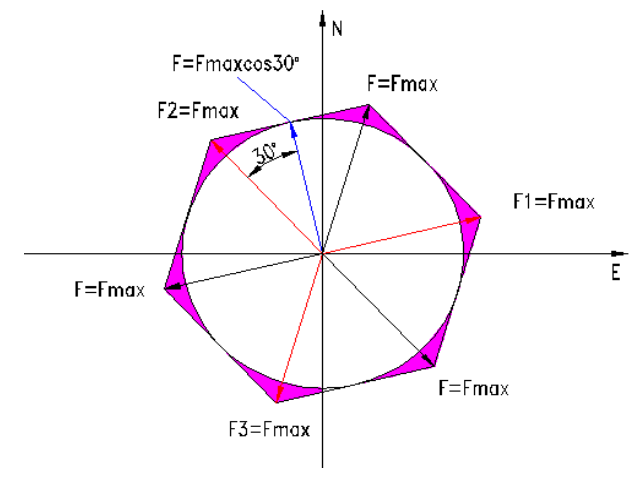

Figure 6. The maximum force of the biasing force vectors using

$$
\text { analytic geometry }
$$

\section{SUMMARY}

In this study, pushes against the stationary rotary steerable systems has the following characteristics:

(1) Using wireless transmission mode for data transmission, data transmission is more accurate, able to get the full MWD measurement data, nearby bit trajectory parameters and downhole working conditions;

(2) Change the trajectory control command parameters into the control command code, achieve to download signal decoding, and passes through the bypass system for data transmission is based on does not affect the mud circulation, the data is accurate; (3) Using two-way communication and power short section is responsible for downhole tools with different data pulse sequence, identification download signal, power generation and pulse control, the design is the first at home and abroad;

(4) In addition, analysts believe that the direction of push force does not have 360 degrees arbitrary adjustable if the resultant force absolute value remains unchanged, it means the area between the inner and outer circle is called 'control dead zone', which should be avoided in the engineering design and directional drilling selection biasing force resultant vector.

In addition, after the completion of the entire assembly of rotary steerable systems mechanical structure design, processing, electronic circuit design, processing, preparation software, assembly tools, the indoors 
experiments laboratory need to be studied, mainly including the guiding actuating mechanism indoor FBI test, laboratory test of guidance control system, non-contact transmission device of laboratory test, To determine the rotary steering system of each subsystem can work, whether can achieve the expected design requirements.

\section{REFERENCES}

[1] Nathan Wicks, Jahir Pabon, Francois Auzerais, Roman Kats, Matthew Godfrey, Yong Chang, Andrew Zheng. Modeling of Axial Vibrations to Allow Intervention in Extended Reach Wells. SPE 156017 at the SPE Deepwater Drilling and Completions Conference held in Galveston, Texas, USA, 20-21 June 2012.

[2] Andereassen E, Blikra H, Hjelle A, etal. RCLS Improves Reservoir Drilling Efficiency and Wellbore Placement. SPE39329.

[3] John A. Using Rotary Closed-Loop Drilling to Increase Operational Efficiency and Reduse Operational Risk. Offshore Europe, 4-7 September, 2001,Aberdeen, United Kingdom.
[4] Reich M, Oesterberg M, Montes H. Straight down to success: performance review of a vertical drilling system, SPE, 2003: 84451.

[5] Su Yinao, Summery and research development on the geometric steerable drilling technology[J]. Petroleum development and exploitation. 2005, (01).

[6] Pang Yuye, Fu Yongsheng, Signal Processing of MWD System [J].Electronic Measurement Technology;2005-02

[7] Zhang Xinhong, Feng Ding(Yangtze University,Jingzhou 434023);Analysis on Structural Deflecting Characters of Downhole Assembly for Steering Drilling[J];Journal of Oil and Gas Technology;2007-02

[8] Li Hanxing (Research Center of CNOOC,Beijing),Jiang Wei Jiang Shiquan.;Development and in-situ test of eccentricity controller for rotary steering drilling.[A];[C];2007.

[9] Liu Xinhua ,Dong Guanghua ,Zhao Hongshan ,Yang Quanjin (Drilling Technology Research Institute,Shengli Petroleum Administration,Sinopec,Dongying,Shandong,257017,China);Desig $\mathrm{n}$ and Laboratory Test of Control System for Rotary Steering Motor[J];Petroleum Drilling Techniques;2011-05.

[10] Peng Yong, Yang Wenhui, Li Junqinag. Petroleum Machinory.Analysis of thrust force of actuating mechanism on rotary steering drilling system[J];2005-11. 